MATHEMATICS OF COMPUTATION

Volume 71, Number 237, Pages 333-342

S 0025-5718(01)01358-8

Article electronically published on June 14, 2001

\title{
SECURITY OF THE MOST SIGNIFICANT BITS OF THE SHAMIR MESSAGE PASSING SCHEME
}

\author{
MARIA ISABEL GONZÁLEZ VASCO AND IGOR E. SHPARLINSKI
}

\begin{abstract}
Boneh and Venkatesan have recently proposed a polynomial time algorithm for recovering a "hidden" element $\alpha$ of a finite field $\mathbb{F}_{p}$ of $p$ elements from rather short strings of the most significant bits of the remainder modulo $p$ of $\alpha t$ for several values of $t$ selected uniformly at random from $\mathbb{F}_{p}^{*}$. Unfortunately the applications to the computational security of most significant bits of private keys of some finite field exponentiation based cryptosystems given by Boneh and Venkatesan are not quite correct. For the Diffie-Hellman cryptosystem the result of Boneh and Venkatesan has been corrected and generalized in our recent paper. Here a similar analysis is given for the Shamir message passing scheme. The results depend on some bounds of exponential sums.
\end{abstract}

\section{INTRODUCTION}

Let $p$ be an $n$-bit prime and let $\mathbb{F}_{p}$ be a field of $p$ elements.

For integers $s$ and $q \geq 1$ we denote by $(s \operatorname{rem} q)$ the remainder of $s$ on division by $q$. We also use $\log z$ to denote the binary $\log$ arithm of $z>0$.

The Shamir message passing scheme can be described in the following way (see [1], as well as Protocol 12.22 from [9]).

To send a message $m \in[0, p-1]$ from Alice to Bob:

- Alice selects a random $a \in[0, p-2]$ with $\operatorname{gcd}(a, p-1)=1$, computes $A=$ $\left(m^{a}\right.$ rem $\left.p\right)$ and sends $A$ to $B o b$.

- $B o b$ selects a random $b \in a[0, p-2]$ with $\operatorname{gcd}(b, p-1)=1$, computes $B=$ $\left(A^{b} \operatorname{rem} p\right) a$ and sends $B$ to Alice.

- Alice finds $u \in[0, p-2]$ satisfying the congruence $a u \equiv 1(\bmod p-1)$, computes $C=\left(B^{u}\right.$ rem $\left.p\right)$ and sends $C$ to $B o b$.

○ Bob finds $v \in[0, p-2]$ satisfying the congruence $b v \equiv 1(\bmod p-1)$, computes $m=\left(C^{v} \operatorname{rem} p\right)$.

Given a primitive root $g \in \mathbb{F}_{p}$, Boneh and Venkatesan [1] have proposed a method of recovering a "hidden" element $\alpha \in \mathbb{F}_{p}$ from about $n^{1 / 2}$ most significant bits of $\left(\alpha g^{x_{i}} \operatorname{rem} p\right), i=1, \ldots, d$, for $d=\left\lceil 2 n^{1 / 2}\right\rceil$ integers $x_{1}, \ldots, x_{d}$, chosen uniformly and independently at random in the interval $[0, p-2]$. This result has been applied to proving security of reasonably small portions of bits of private keys of several cryptosystems. In particular, Theorem 3 of [1] claims the security of the $\left\lceil n^{1 / 2}\right\rceil+$ $\lceil\log n\rceil$ most significant bits of the message in the Shamir message passing scheme.

Received by the editor May 18, 2000.

2000 Mathematics Subject Classification. Primary 94A60; Secondary 11T23, 11 T71.

Key words and phrases. Shamir message passing scheme, bit security, exponential sums, cryptography. 
Unfortunately the proof of this result is not quite correct because the exponent $x$ of the corresponding multiplier $g^{x}$ (where $g$ will in fact be $m^{b}, m$ and $b$ chosen in the scheme) must satisfy the additional condition $\operatorname{gcd}(b x+1, p-1)=1$; thus $g^{x}$ runs through some special subset of $\mathbb{F}_{p}^{*}$ (even if $g$ is a primitive root) rather than through the whole $\mathbb{F}_{p}^{*}$ and therefore Theorem 1 of [1] does not apply. The proof of Theorem 2 in [1], dealing with security of most significant bits of the Diffie-Hellman key, suffers from a similar problem. In [3] the result of Theorem 1 of [1] has been extended to the case when $g$ is not necessarily a primitive root but an element of multiplicative order $T$, provided that $T \geq p^{1 / 3+\varepsilon}$ for any prime $p$ and $T \geq p^{\varepsilon}$ for almost all $p$. It has also been shown that this statement allows us to close the gap in the proof of Theorem 2 of [1]. Namely it is shown that by having an oracle which computes $\left\lceil n^{1 / 2}\right\rceil+\lceil\log n\rceil$ most significant bits of the private key $\left(g^{a b} \operatorname{rem} p\right)$ from the values of the public keys $A=\left(g^{a} \operatorname{rem} p\right)$ and $B=\left(g^{b} \operatorname{rem} p\right)$ one can construct a probabilistic polynomial time algorithm for computing the whole key $\left(g^{a b} \operatorname{rem} p\right)$ for all pairs $(a, b) \in[0, T-1]^{2}$, where $T$ is the multiplicative order of $g$.

The method of [3] relies on some bounds of exponential sums and results about the distribution of exponential functions in residue classes. Here we use a similar approach to study the bit security of the Shamir message passing scheme.

A survey of similar results for other functions of cryptographic interest has recently been given in 2 .

We denote by $\nu(k)$ the number of distinct prime divisors and by $\varphi(k)$ the Euler function of $k \geq 2$.

Throughout the paper the implied constants in symbols ' $O$ ' may occasionally, where obvious, depend on the small positive parameter $\varepsilon$ and are absolute otherwise; they all are effective and can be explicitly evaluated.

\section{Distribution of EXPONENTIAL FUnCtions MODULO $p$}

As in [3] the following bound of exponential sums plays the central role in our arguments.

Let $\mathbf{e}(z)=\exp (2 \pi i z / p)$.

The following estimate is well known (see the proof of Lemma 2 in [7] or Theorem 8.2 in [11]).

Lemma 2.1. For any element $\vartheta \in \mathbb{F}_{p}$ of multiplicative order $\tau$ the bound

$$
\max _{0 \leq H \leq \tau-1} \max _{\operatorname{gcd}(c, p)=1}\left|\sum_{0 \leq x \leq H} \mathbf{e}\left(c \vartheta^{x}\right)\right|=O\left(p^{1 / 2} \log p\right)
$$

holds.

For $b \in[1, p-2]$ with $\operatorname{gcd}(b, p-1)=1$ we denote by $\mathcal{X}_{b}$ the set of integers $x \in[0, p-2]$ with $\operatorname{gcd}(b x+1, p-1)=1$. In particular, $\# \mathcal{X}_{b}=\varphi(p-1)$.

Let us fix an element $g$ of multiplicative order $T$ modulo $p$. Combining Lemma 2.1] with the sieve of Eratosthenes we derive

Lemma 2.2. For any $b$ with $\operatorname{gcd}(b, p-1)=1$ the bound

$$
\max _{\operatorname{gcd}(c, p)=1}\left|\sum_{x \in \mathcal{X}_{b}} \mathbf{e}\left(c g^{x}\right)\right|=O\left(2^{\nu(p-1)} p^{1 / 2} \log p\right)
$$

holds. 
Proof. Let $\mu(k)$ denote the Möbius function. We recall that $\mu(1)=1, \mu(k)=0$ if $k \geq 2$ is not square-free and $\mu(k)=(-1)^{\nu(k)}$ otherwise.

Using the Möbius function $\mu(d)$ over the divisors of $p-1$ to detect the co-primality condition and interchanging the order of summation, we obtain (see Section 3.d of Chapter 2 of [14])

$$
\sum_{x \in \mathcal{X}_{b}} \mathbf{e}\left(c g^{x}\right)=\sum_{d \mid p-1} \mu(d) \sum_{\substack{x=0 \\ b x+1 \equiv 0}}^{p-2} \mathbf{e}\left(c g^{x}\right)
$$

Since $\operatorname{gcd}(b, p-1)=1$, the condition $b x+1 \equiv 0(\bmod d)$ can be written in the form $x=d z+\alpha_{d}$ with some integer $\alpha_{d}, 1 \leq \alpha_{d} \leq d-1$. Therefore

$$
\sum_{\substack{x=0 \\ b x+1 \equiv 0}}^{p-2} \mathbf{e}_{p}\left(c g^{x}\right)=\sum_{0 \leq d z+\alpha_{d} \leq p-2} \mathbf{e}_{p}\left(c g^{d z+\alpha_{d}}\right) .
$$

Denoting by $\tau_{d}$ the multiplicative order of $\vartheta_{d}=g^{d}$ and remarking that $\tau_{d} \geq T / d$, we derive from Lemma 2.1

$$
\begin{aligned}
\sum_{0 \leq d z+\alpha_{d} \leq p-2} \mathbf{e}_{p}\left(c g^{d z+\alpha_{d}}\right) & =\sum_{0 \leq z \leq\left(p-2-\alpha_{d}\right) / d} \mathbf{e}_{p}\left(c g^{\alpha_{d}} g_{d}^{z}\right) \\
& =O\left(\left(\left\lfloor\frac{p-1}{\tau_{d} d}\right\rfloor+1\right) p^{1 / 2} \log p\right) \\
& =O\left(\left(\left\lfloor\frac{p-1}{T}\right\rfloor+1\right) p^{1 / 2} \log p\right) \\
& =O\left(p^{3 / 2} T^{-1} \log p\right) .
\end{aligned}
$$

Taking into account that

$$
\sum_{d \mid p-1}|\mu(d)|=2^{\nu(p-1)}
$$

(see Section 3.b of Chapter 2 of [14]), we obtain the desired result.

For integers $\lambda, b, r$ and $h$ let us denote by $N_{\lambda, b}(r, h)$ the number of $x \in \mathcal{X}_{b}$ with $\left(\lambda g^{x} \operatorname{rem} p\right) \in[r+1, r+h]$.

We need the following asymptotic formula which shows that $N_{\lambda, b}(r, h)$ is close to its expected value $\varphi(p-1) h / p$.

Lemma 2.3. For any $\varepsilon>0$ there exists $\delta>0$ such that for $T \geq p^{1 / 2+\varepsilon}$ the bound

$$
\max _{0 \leq r, h \leq p-1} \max _{\operatorname{gcd}(\lambda, p)=1} \max _{\operatorname{gcd}(b, p-1)=1}\left|N_{\lambda, b}(r, h)-\frac{\varphi(p-1) h}{p}\right|=O\left(p^{1-\delta}\right)
$$

holds.

Proof. We remark that $N_{\lambda, b}(r, h)$ is the number of solutions of the congruence

$$
\lambda g^{x} \equiv y \quad(\bmod p), \quad x \in \mathcal{X}_{b}, y=r+1, \ldots, r+h .
$$

Using the identity (see Exercise 11.a in Chapter 3 of [14])

$$
\sum_{c=0}^{p-1} \mathbf{e}(c u)=\left\{\begin{array}{lll}
0, & \text { if } u \neq 0 & (\bmod p) \\
p, & \text { if } u \equiv 0 & (\bmod p)
\end{array}\right.
$$


we obtain

$$
\begin{aligned}
N_{\lambda, b}(r, h) & =\frac{1}{p} \sum_{x \in \mathcal{X}_{b}} \sum_{y=r+1}^{r+h} \sum_{c=0}^{p-1} \mathbf{e}\left(c\left(\lambda g^{x}-y\right)\right) \\
& =\frac{1}{p} \sum_{c=0}^{p-1} \sum_{x \in \mathcal{X}_{b}} \mathbf{e}\left(c \lambda g^{x}\right) \sum_{y=r+1}^{r+h} \mathbf{e}(-c y) .
\end{aligned}
$$

Separating the term $\# \mathcal{X}_{b} h / p=\varphi(p-1) h / p$ corresponding to $c=0$ we obtain

$$
\begin{aligned}
\left|N_{\lambda, b}(r, h)-\frac{N_{b} h}{p}\right| & \leq \frac{1}{p} \sum_{c=1}^{p-1}\left|\sum_{x \in \mathcal{X}_{b}} \mathbf{e}\left(c \lambda g^{x}\right)\right|\left|\sum_{y=r+1}^{r+h} \mathbf{e}(-c y)\right| \\
& =\frac{1}{p} \sum_{c=1}^{p-1}\left|\sum_{x \in \mathcal{X}_{b}} \mathbf{e}\left(c \lambda g^{x}\right)\right|\left|\sum_{y=r+1}^{r+h} \mathbf{e}(c y)\right|
\end{aligned}
$$

Using Lemma 2.2 and the estimate

$$
\max _{0 \leq r, h \leq p-1} \sum_{c=1}^{p-1}\left|\sum_{y=r+1}^{r+h} \mathbf{e}(c y)\right|=O(p \log p)
$$

(see Exercise 11.c in Chapter 3 of [14]), we obtain

$$
\left|N_{\lambda, b}(r, h)-\frac{\varphi(p-1) h}{p}\right|=O\left(2^{\nu(p-1)} p^{3 / 2} T^{-1} \log ^{2} p\right) .
$$

Because $\nu(p-1) ! \leq p-1$, we conclude that $2^{\nu(p-1)} \leq p^{o(1)}$ and the desired result follows.

\section{LATtices}

As in [1, our results rely on rounding techniques in lattices. We therefore review a few related results and definitions.

Let $\left\{\mathbf{b}_{1}, \ldots, \mathbf{b}_{s}\right\}$ be a set of linearly independent vectors in $\mathbb{R}^{s}$. The set of vectors

$$
L=\left\{\mathbf{z}: \mathbf{z}=\sum_{i=1}^{s} t_{i} \mathbf{b}_{i}, \quad t_{1}, \ldots, t_{s} \in \mathbb{Z}\right\}
$$

is called an $s$-dimensional full rank lattice. The set $\left\{\mathbf{b}_{1}, \ldots, \mathbf{b}_{s}\right\}$ is called the basis of $L$.

It has been remarked in Section 2.1 of [8] and then in Section 2.4 of [10] that the following statement holds which is somewhat stronger than that usually used in the literature.

Lemma 3.1. There exists a polynomial time algorithm which, for a given lattice $L$ and a vector $\mathbf{r}=\left(r_{1}, \ldots, r_{s}\right) \in \mathbb{R}^{s}$, finds a lattice vector $\mathbf{v}=\left(v_{1}, \ldots, v_{s}\right)$ satisfying the inequality

$$
\begin{aligned}
& \sum_{i=1}^{s}\left(v_{i}-r_{i}\right)^{2} \\
& \quad \leq \exp \left(O\left(\frac{s \log ^{2} \log s}{\log s}\right)\right) \min \left\{\sum_{i=1}^{s}\left(z_{i}-r_{i}\right)^{2}, \quad \mathbf{z}=\left(z_{1}, \ldots, z_{s}\right) \in L\right\} .
\end{aligned}
$$


Proof. The statement is a combination of the Schnorr modification [13] of the lattice basis reduction algorithm of Lenstra, Lenstra and Lovász [5] with a result of Kannan [6] about reduction of the closest vector problem to the shortest vector problem.

For integers $g$ and $x_{1}, \ldots, x_{d}$, selected in the interval $[0, p-2]$, we denote by $L_{g, p}\left(x_{1}, \ldots, x_{d}\right)$ the $(d+1)$-dimensional lattice generated by the rows of the following $(d+1) \times(d+1)$-matrix:

$$
\left(\begin{array}{llllll}
p & 0 & 0 & \ldots & 0 & 0 \\
0 & p & 0 & \ldots & 0 & 0 \\
& \vdots & & & & \vdots \\
0 & 0 & 0 & \ldots & p & 0 \\
t_{1} & t_{2} & t_{3} & \ldots & t_{d} & 1 / p
\end{array}\right)
$$

where $t_{i}=\left(g^{x_{i}} \operatorname{rem} p\right), i=1, \ldots, d$.

The following result is a generalization of Theorem 5 of [1] (which corresponds to the case $T=p-1)$.

Lemma 3.2. Let $d=2\left\lceil n^{1 / 2}\right\rceil$ and $\mu=n^{1 / 2} / 2+3$, p sufficiently large prime number. Let $\alpha$ be a fixed integer in the interval $[0, p-1]$. For any $\varepsilon>0$, any element $g \in \mathbb{F}_{p}$ of multiplicative order $T \geq p^{1 / 2+\varepsilon}$ and any $b \in[1, p-2]$ with $\operatorname{gcd}(b, p-1)=1$ the following statement holds: Chosen integers $x_{1}, \ldots, x_{d}$ uniformly and independently at random in the set $\mathcal{X}_{b}$, then with probability $P \geq$ $1-2^{-n^{1 / 2}}$ for any vector $\mathbf{u}=\left(u_{1}, \ldots, u_{d}, 0\right)$ with

$$
\left(\sum_{i=1}^{d}\left(\left(\alpha g^{x_{i}} \operatorname{rem} p\right)-u_{i}\right)^{2}\right)^{1 / 2} \leq p 2^{-\mu}
$$

all vectors $\mathbf{v}=\left(v_{1}, \ldots, v_{d}, v_{d+1}\right) \in L_{g, p}\left(x_{1}, \ldots, x_{d}\right)$ satisfying

$$
\left(\sum_{i=1}^{d}\left(v_{i}-u_{i}\right)^{2}\right)^{1 / 2} \leq p 2^{-\mu}
$$

are of the form

$$
\mathbf{v}=\left(\left(\beta g^{x_{1}} \operatorname{rem} p\right), \ldots,\left(\beta g^{x_{d}} \operatorname{rem} p\right), \beta / p\right)
$$

with some $\beta \equiv \alpha(\bmod p)$.

Proof. As in [1] we define the modular distance between two integers $\beta$ and $\gamma$ as

$$
\operatorname{dist}_{p}(\beta, \gamma)=\min _{b \in \mathbb{Z}}|\beta-\gamma-b p|=\min \{((\beta-\gamma) \operatorname{rem} p), p-((\beta-\gamma) \operatorname{rem} p)\} \text {. }
$$

It follows from Lemma 2.3 that for any $\beta$ and $\gamma$ such that $\beta \not \equiv \gamma(\bmod p)$ the probability $P(\beta, \gamma)$ of

$$
\operatorname{dist}_{p}\left(\beta g^{x}, \gamma g^{x}\right)>p 2^{-\mu+1}
$$

for an integer $x$ chosen uniformly at random in the set $\mathcal{X}_{b}$ is

$$
P(\beta, \gamma)=1-2^{-\mu+2}+O\left(p^{-\delta}\right)
$$

for some $\delta>0$, depending only on $\varepsilon$. Thus

$$
P(\beta, \gamma) \geq 1-\frac{5}{2^{\mu}}
$$


provided that $p$ is large enough.

Therefore, for any $\beta \not \equiv \alpha(\bmod p)$,

$\operatorname{Pr}\left[\exists i \in[1, d] \mid \operatorname{dist}_{p}\left(\beta g^{x_{i}}, \alpha g^{x_{i}}\right)>p 2^{-\mu+1}\right]=1-(1-P(\alpha, \beta))^{d} \geq 1-\left(\frac{5}{2^{\mu}}\right)^{d}$,

where the probability is taken over integers $x_{1}, \ldots, x_{d}$ chosen uniformly and independently at random in the set $\mathcal{X}_{b}$.

Since for $\beta \not \equiv \alpha(\bmod p)$ there are only $p-1$ possible values for $(\beta \operatorname{rem} p)$, we obtain

$$
\begin{aligned}
& \operatorname{Pr}\left[\forall \beta \not \equiv \alpha \quad(\bmod p), \exists i \in[1, d] \mid \operatorname{dist}_{p}\left(\beta g^{x_{i}}, \alpha g^{x_{i}}\right)>p 2^{-\mu+1}\right] \\
& \geq 1-(p-1)\left(\frac{5}{2^{\mu}}\right)^{d}>1-2^{-n^{1 / 2}}
\end{aligned}
$$

because

$$
d(\mu-\log 5)>\left\lceil n^{1 / 2}\right\rceil n^{1 / 2}+2\left\lceil n^{1 / 2}\right\rceil(3-\log 5)>\log p+n^{1 / 2} .
$$

The rest of the proof is identical to the proof of Theorem 5 of [1]; we outline it for the sake of completeness.

Let us fix some integers $x_{1}, \ldots, x_{d}$ with

$$
\min _{\beta \neq \alpha} \min _{(\bmod p)} \operatorname{mist}_{i \in[1, d]}\left(\beta g^{x_{i}}, \alpha g^{x_{i}}\right)>p 2^{-\mu+1} .
$$

Let $\mathbf{v}$ be a lattice point satisfying

$$
\left(\sum_{i=1}^{d}\left(v_{i}-u_{i}\right)^{2}\right)^{1 / 2} \leq p 2^{-\mu} .
$$

Clearly, since $\mathbf{v} \in L_{g, p}\left(x_{1}, \ldots, x_{d}\right)$, there are integers $\beta, z_{1}, \ldots, z_{d}$ such that

$$
\mathbf{v}=\left(\beta t_{1}-z_{1} p, \ldots, \beta t_{d}-z_{d} p, \beta / p\right)
$$

where, as in (3.1), $t_{i}=\left(g^{x_{i}} \operatorname{rem} p\right), i=1, \ldots, d$.

If $\beta \equiv \alpha(\bmod p)$, then for all $i=1, \ldots, d$ we have $\beta t_{i}-z_{i} p=\left(\beta t_{i} \operatorname{rem} p\right)$, for otherwise there would be $j \in\{1, \ldots, d\}$ so that $\left|v_{j}-u_{j}\right|>p 2^{-\mu}$.

Now suppose that $\beta \not \equiv \alpha(\bmod p)$. In this case we have

$$
\begin{aligned}
\left(\sum_{i=1}^{d}\left(v_{i}-u_{i}\right)^{2}\right)^{1 / 2} & \geq \min _{i \in[1, d]} \operatorname{dist}_{p}\left(\beta t_{i}, u_{i}\right) \\
& \geq \min _{i \in[1, d]}\left(\operatorname{dist}_{p}\left(\beta t_{i}, \alpha t_{i}\right)-\operatorname{dist}_{p}\left(u_{i}, \alpha t_{i}\right)\right) \\
& >p 2^{-\mu+1}-p 2^{-\mu}=p 2^{-\mu},
\end{aligned}
$$

which contradicts our assumption. As we have seen, condition (3.2) holds with probability exceeding $1-2^{-n^{1 / 2}}$ and the result follows.

For an integer $k \geq 1$ we define $f_{k}(t)$ by the inequalities

$$
\left(f_{k}(t)-1\right) \frac{p}{2^{k}} \leq(t \operatorname{rem} p)<f_{k}(t) \frac{p}{2^{k}} .
$$

Thus, roughly speaking, $f_{k}(t)$ is the integer defined by the $k$ most significant bits of $(t \operatorname{rem} p)$.

Using Lemma 3.2 in the same way as Theorem 5 of [1] is used in the proof of Theorem 1, we obtain 
Lemma 3.3. Let $d=2\left\lceil n^{1 / 2}\right\rceil$ and $k=\left\lceil n^{1 / 2}\right\rceil+\lceil\log n\rceil$. For any $\varepsilon>0$, any element $g \in \mathbb{F}_{p}$ of multiplicative order $T \geq p^{1 / 2+\varepsilon}$ and any $b \in[1, p-2]$ with $\operatorname{gcd}(b, p-1)=1$ the following statement holds: There exists a deterministic polynomial time algorithm $\mathcal{A}$ such that for any integer $\alpha \in[1, p-1]$ given $2 d$ integers

$$
t_{i}=\left(g^{x_{i}} \operatorname{rem} p\right) \quad \text { and } \quad s_{i}=f_{k}\left(\alpha t_{i}\right), \quad i=1, \ldots, d,
$$

its output satisfies

$$
\operatorname{Pr}_{x_{1}, \ldots, x_{d} \in \mathcal{X}_{b}}\left[\mathcal{A}\left(t_{1}, \ldots, t_{d} ; s_{1}, \ldots, s_{d}\right)=\alpha\right] \geq 1-2^{-n^{1 / 2}}
$$

if $x_{1}, \ldots, x_{d}$ are chosen uniformly and independently at random in the set $\mathcal{X}_{b}$.

Proof. We follow the same arguments as in the proof of Theorem 1 in 1$]$ which we briefly outline here for the sake of completeness. We refer to the first $d$ vectors in the defining matrix of $L_{g, p}\left(x_{1}, \ldots, x_{d}\right)$ as $p$-vectors.

Let us consider the vector $\mathbf{r}=\left(r_{1}, \ldots, r_{d}, r_{d+1}\right)$ where

$$
r_{i}=s_{i} \frac{p}{2^{k}}, \quad i=1, \ldots, d, \quad \text { and } \quad r_{d+1}=0 .
$$

Multiplying the last row vector $\left(t_{1}, \ldots, t_{d}, 1 / p\right)$ of the matrix (3.1) by $\alpha$ and subtracting certain multiples of $p$-vectors, we obtain a lattice point

$$
\mathbf{u}_{\alpha}=\left(u_{1}, \ldots, u_{d}, \alpha / p\right) \in L_{g, p}\left(x_{1}, \ldots, x_{d}\right)
$$

such that

$$
\left|u_{i}-r_{i}\right|<p 2^{-k}, \quad i=1, \ldots, d
$$

Therefore,

$$
\left(\sum_{i=1}^{d+1}\left(u_{i}-r_{i}\right)^{2}\right)^{1 / 2} \leq p(d+1)^{1 / 2} 2^{-k} .
$$

Now we can use Lemma 3.1 (with a slightly rougher constant $2^{(d+1) / 4}$ ) to find in polynomial time a lattice vector $\mathbf{v}=\left(v_{1}, \ldots, v_{d}, v_{d+1}\right) \in L_{g, p}\left(x_{1}, \ldots, x_{d}\right)$ such that

$$
\begin{aligned}
& \left(\sum_{i=1}^{d}\left(v_{i}-r_{i}\right)^{2}\right)^{1 / 2} \\
& \quad \leq 2^{(d+1) / 4} \min \left\{\left(\sum_{i=1}^{d+1}\left(z_{i}-r_{i}\right)^{2}\right)^{1 / 2}, \quad \mathbf{z}=\left(z_{1}, \ldots, z_{d}, z_{d+1}\right) \in L\right\} \\
& \quad \leq 2^{(d+1) / 4} p(d+1)^{1 / 2} 2^{-k} \leq p 2^{-\mu-1},
\end{aligned}
$$

where $\mu=n^{1 / 2} / 2+3$, provided that $n$ is sufficiently large. We also have

$$
\left(\sum_{i=1}^{d}\left(u_{i}-r_{i}\right)^{2}\right)^{1 / 2} \leq p d^{1 / 2} 2^{-k} \leq p 2^{-\mu-1} .
$$

Therefore,

$$
\left(\sum_{i=1}^{d}\left(u_{i}-v_{i}\right)^{2}\right)^{1 / 2} \leq p 2^{-\mu}
$$


Applying Lemma 3.2, we see that $\mathbf{v}=\mathbf{u}_{\alpha}$ with probability at least $1-2^{-n^{1 / 2}}$, and therefore, $\alpha$ can be recovered in polynomial time.

\section{Security of the most significant Bits of the Shamir scheme}

We are ready to prove the main results.

For a positive integer $k$ we suppose that we are given an oracle $\mathcal{O}_{k}$ such that for any given values of $A, B, C$ it outputs the $k$ most significant bits of $m$ if the triple $(A, B, C)$ corresponds to a proper usage of the Shamir message passing scheme and an error message, otherwise.

More precisely, given $A, B$ and $C$, the oracle $\mathcal{O}_{k}$ outputs:

$\circ f_{k}(m)$, if

$$
A=\left(m^{a} \operatorname{rem} p\right), \quad B=\left(A^{b} \operatorname{rem} p\right), \quad C=\left(B^{u} \operatorname{rem} p\right),
$$

where $a u \equiv 1(\bmod p-1)$ for some $m \in[1, p-1]$ and $a, b \in[0, p-2]$ with $\operatorname{gcd}(a b, p-1)=1$;

$\circ$ an error message, otherwise.

Theorem 4.1. Assume that we are given an oracle $\mathcal{O}_{k}$ as above, with

$$
k=\left\lceil n^{1 / 2}\right\rceil+\lceil\log n\rceil .
$$

Then there exists a probabilistic polynomial time algorithm which computes the message $m$, for all except $O\left(p^{1 / 2+\varepsilon}\right)$ messages $m \in[1, p-1]$, from the values of $A=\left(m^{a} \operatorname{rem} p\right), B=\left(A^{b} \operatorname{rem} p\right)$ and $C=\left(B^{u} \operatorname{rem} p\right)$, where $a, b \in[0, p-2]$ with $\operatorname{gcd}(a b, p-1)=1$ and $a u \equiv 1(\bmod p-1)$, which uses the expected number of $O\left(n^{1 / 2} \log n\right)$ calls of the oracle $\mathcal{O}_{k}$.

Proof. We exclude from the consideration the messages $m \in[1, p-1]$ of multiplicative order less than $p^{1 / 2+\varepsilon}$. Obviously, the number $E$ of such excluded messages does not exceed

$$
E \leq \tau(p-1) p^{1 / 2+\varepsilon / 2},
$$

where $\tau(p-1)$ is the number of positive integer divisors of $p-1$. Indeed, for any divisor $D \mid p-1$ there are at most $D$ values of $m \in[1, p-1]$ of multiplicative order $D$. Using the bound $\tau(p-1)=O\left(p^{\varepsilon / 2}\right)$ (see Theorem 5.2 of Chapter 1 of [12]), we obtain from (4.1) that the exceptional set is of size $E=O\left(p^{1 / 2+\varepsilon}\right)$.

Let us consider a message $m$ of multiplicative order $T \geq p^{1 / 2+\varepsilon / 2}$.

For $x \in \mathcal{X}_{b}$ we put $a_{x}=a$ and define $b_{x} \in[1, p-2]$ from the congruence

$$
b_{x}(b x+1) \equiv b \quad(\bmod p-1) .
$$

We also put

We remark that

$$
m_{x}=\left(m^{1+b x} \operatorname{rem} p\right)
$$

$$
A_{x}=\left(m_{x}^{a_{x}} \operatorname{rem} p\right), \quad B_{x}=\left(m_{x}^{a_{x} b_{x}} \operatorname{rem} p\right), \quad C_{x}=\left(m_{x}^{b_{x}} \operatorname{rem} p\right)
$$

can be computed as

$$
A_{x}=\left(A C^{x} \operatorname{rem} p\right), \quad B_{x}=B, \quad C_{x}=C .
$$

Although the value of $b$ is not known, one can select elements $x \in \mathcal{X}_{b}$ uniformly and independently at random by querying the oracle $\mathcal{O}_{k}$ with the triples 
$\left(A_{x}, B_{x}, C_{x}\right)$ where the elements $x$ are selected uniformly and independently at random in the interval $[0, p-2]$. If $\operatorname{gcd}(b x+1, p-1)>1$, the oracle returns an error message; otherwise $x \in \mathcal{X}_{b}$. Now we choose $d=2\left\lceil n^{1 / 2}\right\rceil$ elements $x_{1}, \ldots, x_{d} \in \mathcal{X}_{b}$ uniformly and independently at random. Because

$$
\frac{p-1}{\# \mathcal{X}_{b}}=\frac{p-1}{\varphi(p-1)}=O(\log \log p)
$$

(see Theorem 5.1 of Chapter 1 of [12]), we see that the expected number of choices of $x \in[0, p-2]$ before we get $d$ elements in $\mathcal{X}_{b}$ is $O(d \log \log p)=O\left(n^{1 / 2} \log n\right)$. We remark that these elements are independent and uniformly distributed in $\mathcal{X}_{b}$. Moreover, every output of the oracle provides $k$ most significant bits of $m_{x}$. Remarking that $m_{x} \equiv m C^{x}(\bmod p)$ and that $C \equiv m^{b}(\bmod p)$ is of multiplicative order $T$ (because $\operatorname{gcd}(b, p-1)=1$ ), we see that Lemma 3.3 applies and the result follows.

\section{REMARKS}

First of all we note that the constants in the above estimates are effective and can be explicitly evaluated.

We have not used the full power of Lemma 3.1 but rather we have applied it with the same constant as in [1]. It is easy to see that in fact the results of [1] as well as our results hold with some

$$
k=O\left(\frac{n^{1 / 2} \log \log n}{\log ^{1 / 2} n}\right)
$$

and a slightly large number of oracle calls.

We also remark that one can consider an oracle which instead of returning an error message for "inconsistent" inputs $(A, B, C)$ returns just a random element from $\mathbb{F}_{p}$. In this case repeating each query twice one can easily distinguish between an $x \in \mathcal{X}_{b}$ and other values.

\section{ACKNOWLEDGMENTS}

We thank Consuelo Martínez for her interest and for helpful advice. We also thank Mats Näslund for fruitful discussions.

\section{REFERENCES}

[1] D. Boneh and R. Venkatesan, Hardness of computing the most significant bits of secret keys in Diffie-Hellman and related schemes, Lect. Notes in Comp. Sci., Springer-Verlag, Berlin, 1109 (1996), 129-142.

[2] M. I. González Vasco and M. Näslund, A survey of hard core functions, Proc. Workshop on Cryptography and Computational Number Theory, Singapore 1999, Birkhäuser, 2001, 227-256.

[3] M. I. González Vasco and I. E. Shparlinski, On the security of Diffie-Hellman bits, Proc. Workshop on Cryptography and Computational Number Theory, Singapore 1999, Birkhäuser, 2001, 257-268.

[4] S. V. Konyagin and I. E. Shparlinski, Character sums with exponential functions and their applications, Cambridge Univ. Press, Cambridge, 1999. MR 2000h:11089

[5] A. K. Lenstra, H. W. Lenstra and L. Lovász, Factoring polynomials with rational coefficients, Mathematische Annalen, 261 (1982), 515-534. MR 84a:12002

[6] R. Kannan, Algorithmic geometry of numbers, Annual Review of Comp. Sci., 2 (1987), 231267. MR 89a:11131

[7] N. M. Korobov, 'On the distribution of digits in periodic fractions', Math. USSR - Sbornik, 18 (1972), 659-676. MR 59:12619] 
[8] D. Micciancio, On the hardness of the shortest vector problem, PhD Thesis, MIT, 1998.

[9] A. J. Menezes, P. C. van Oorschot and S. A. Vanstone, Handbook of Applied Cryptography, CRC Press, Boca Raton, FL, 1997. MR 99g:94015

[10] P. Nguyen and J. Stern, Lattice reduction in cryptology: An update, Lect. Notes in Comp. Sci., Springer-Verlag, Berlin, 1838 (2000), 85-112.

[11] H. Niederreiter, Quasi-Monte Carlo methods and pseudo-random numbers, Bull. Amer. Math. Soc., 84 (1978), 957-1041. MR 80d:65016

[12] K. Prachar, Primzahlverteilung, Springer-Verlag, Berlin, 1957. MR 19:393b

[13] C. P. Schnorr, A hierarchy of polynomial time basis reduction algorithms, Theor. Comp. Sci., 53 (1987), 201-224. MR 89h:11085

[14] I. M. Vinogradov, Elements of number theory, Dover Publ., New York, 1954. MR 19:933e

Department of Mathematics, University of Oviedo, Oviedo, 33007, Spain

E-mail address: mvasco@orion.ciencias.uniovi.es

Dept. of Computing, Macquarie University, Sydney, NSW 2109, Australia

E-mail address: igor@ics.mq.edu.au 